\title{
Review of results of a Visual Analogue Scale (VAS) as a Patient Related Outcome Measure (PROM) for one surgeon's septorhinoplasty series over an eight year period
}

H Lancer, Walsall Hospitals NHS Trust

M Simmons, Walsall Hospitals NHS Trust \& University Hospitals Birmingham NHS Foundation Trust

\section{Background}

Clinical Commissioning Groups (CCGs) are seeking to restrict certain NHS surgical procedures on financial grounds by deeming them 'Procedures of Limited Clinical Value' (PLCV). Septorhinoplasty is one of these. Without access to this procedure, patients may suffer and trainees will become deskilled.

The Royal College of Surgeons (RCS) state that 'many proven operations known to enhance health and improve quality of life have been included in this category (PLCV), and hence are being denied to patients who need them'. They go on to state that procedures which improve 'quality of life' should remain core NHS activity. ${ }^{1}$

Furthermore, the RCS, alongside the GMC, states that for cosmetic procedures, as clinicians 'you must seek and act on feedback from patients, including information on their satisfaction and physical and psychological outcomes. You must use this to inform your practice and improve the quality of your work'. 2

Patient Recorded Outcome Measures (PROMs) were introduced by the Department of Health in April 2009 for four key surgical procedures (hip and knee replacement, varicose vein surgery, hernia repair and cataract surgery), and the use of PROMs has been included in the NHS Outcomes Framework since April 2011. They aim to assess the quality of care from the patients' point of view.

We show how PROMS can be applied to septorhinoplasty surgery in order to illustrate improvement in patients' quality of life.

\section{Objectives}

To document and measure the cosmetic and functional outcomes of this procedure, to aid quality assurance, and help validate this operation as a legitimate NHS procedure.

\section{Methods}

- A 100mm visual analogue scale (VAS) was used to collect both cosmetic and functional PROM data prospectively on 302 consecutive patients for one surgeon's septorhinoplasty series over a period of eight years (between September 2009 and September 2017). (Figure1)

- VAS data was collected pre-operatively and at both 3 and 12 months post-operatively.

- All procedures were supervised or performed by a single consultant.

$\begin{array}{lcc}\text { Cosmesis } & \text { (bad) } 0 & 100 \text { (good) } \\ \text { Airway Left } & 0 & 100 \\ \text { Airway Right } & 0 & 100\end{array}$

Figure 1
A marked improvement for both function and cosmesis was reported post-operatively.

-Of the 302 patients, 174 had post-operative data at 3 months, and 99 had post-operative data at 12 months.

-189 cases had pre-operative data and either 3 or 12 month post-operative data.

-74 patients had a full data set (pre-operative, 3 months postoperatively and 12 months post-operatively). This was analysed initially. (Figure 2)

-There was no significant difference between VAS improvement scores at 3 and 12 months.

-The pre-operative VAS data was then compared with the longest VAS data available (12 month data where available, or 3 month data if the patient then defaulted from further follow up). (Figure 3)

\section{Figure $2 \quad$ Mean Pre- and Post-operative VAS scores for Cosmesis and Function (full data set $n=74$ )}

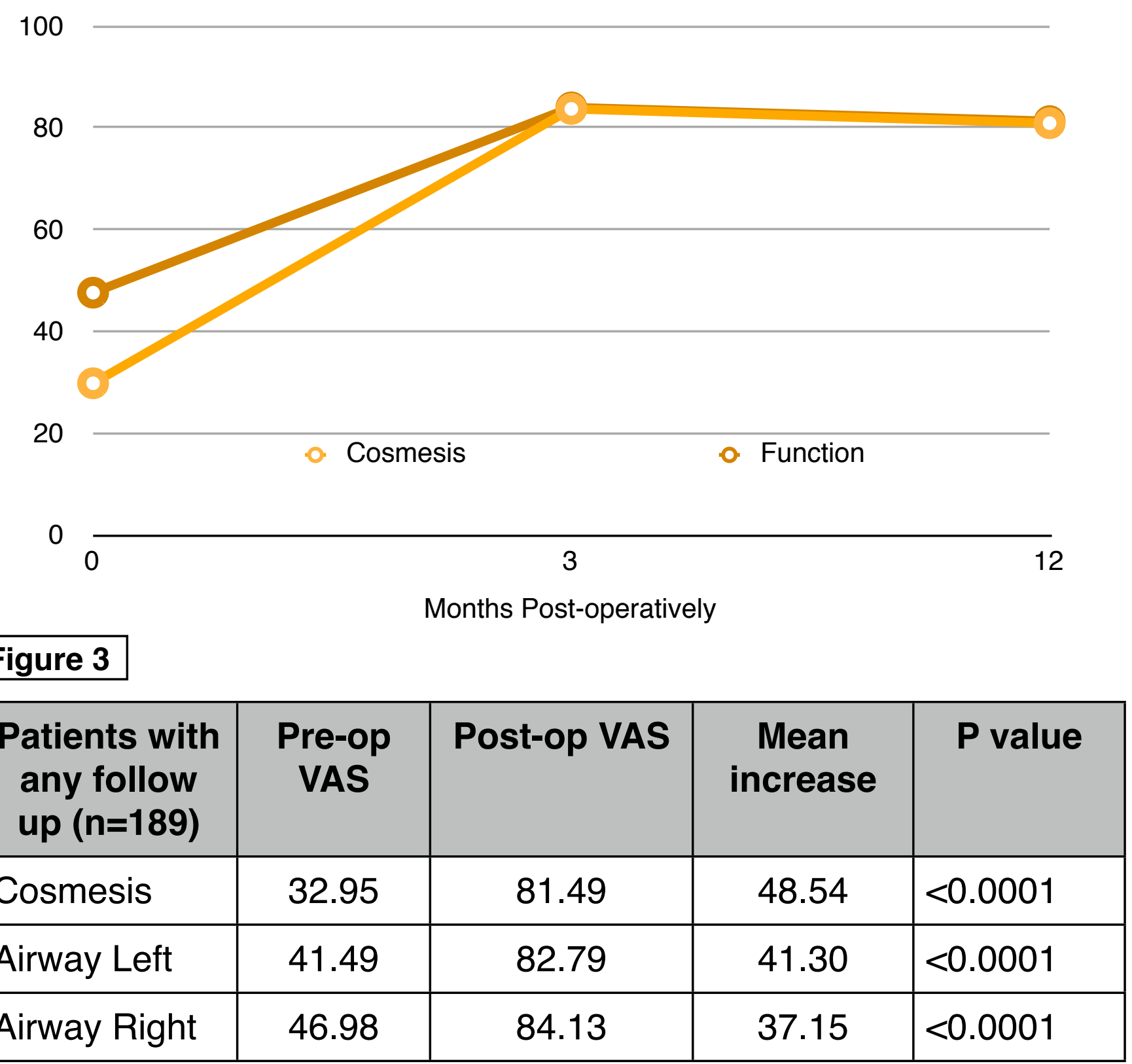

\section{Conclusion}

A simple 3 point VAS can be used to document both airway function and cosmesis pre- and post-operatively. Clear and lasting improvements in both airway and cosmesis have been demonstrated following septorhinoplasty in this series and this helps validate this procedure as a legitimate and useful NHS operation.

\section{References}

1. Procedures of limited clinical value. 2011.The Royal College of Surgeons. [Accessed online February 2018]

2. Professional standards for cosmetic surgery. 2016 The Royal College of Surgeons. [Accessed online February 2018] 